\title{
Correlation Between Thymoma and Soluble Interleukin-2 Receptor Expression in a Patient with Good Syndrome
}

\author{
Haruka Kitano \\ Fumihiro Yamaguchi iD \\ Kenji Atarashi \\ Mina Hiraiwa \\ Yo Shiratori \\ Shota Onozaki \\ Yusuke Shikama
}

Department of Respiratory Medicine, Showa University Fujigaoka Hospital, Yokohama, Kanagawa, 227-850I, Japan
Correspondence: Fumihiro Yamaguchi Department of Respiratory Medicine, Showa University Fujigaoka Hospital, I-30 Fujigaoka, Aoba-ku, Yokohama, Kanagawa, 227-850 I, Japan

Tel +8I-45-973-2848

Email f_y@med.showa-u.ac.jp

\begin{abstract}
Good syndrome is a rare condition characterized by the presence of thymoma in combination with adult-onset hypogammaglobulinemia. Immunological features of Good syndrome include various immunodeficiencies accompanied with hypogammaglobulinemia. In patients with thymoma, paraneoplastic syndromes including hypogammaglobulinemia worsen the prognosis. We herein describe a patient with advanced-stage type A thymoma who was effectively treated with chemotherapy and exhibited a parallel decrease in the serum level of soluble interleukin-2 receptor (sIL-2R), which depends on cellular immunity. The present case suggests the efficacy of sIL-2R as a potential prognostic biomarker in a subset of patients with Good syndrome.
\end{abstract}

Keywords: thymoma, hypogammaglobulinemia, Good syndrome, soluble interleukin-2 receptors

\section{Introduction}

Thymoma, a rare thoracic neoplasm that originates from thymic epithelial cells, is a typically slow-growing tumor and spreads by local extension, and extrathoracic lesions are not common. ${ }^{1}$ Staging and histological subtype are the most important prognostic factors, and paraneoplastic syndromes, including hypogammaglobulinemia, worsen prognosis. ${ }^{2,3}$ There are currently no prognostic biomarkers that have been validated for thymoma. Adult-onset hypogammaglobulinemia occurs in $2-5 \%$ of patients with thymoma. ${ }^{4}$ Good syndrome, which was originally described as thymoma accompanied with hypogammaglobulinemia, is currently defined as thymoma with any type of unclassified immunodeficiency beyond hypogammaglobulinemia, including an abnormal lymphocyte count. ${ }^{2,5}$ Here, we describe a case of thymoma with systemic metastasis in a patient with Good syndrome and report the efficacy of soluble interleukin-2 receptor (sIL-2R) level as a potential biomarker for Good syndrome.

\section{Case Report}

A 50-year-old Japanese woman presented with productive cough, dyspnea on effort, and diarrhea. She had no history of compromised immunity. Chest radiograph revealed widening of the mediastinum and bilateral pleural effusion. Positron emission tomography combined with computed tomography (CT) revealed fluorine-18 fluorodeoxyglucose uptake within a mass in anterior mediastinum and lymph 

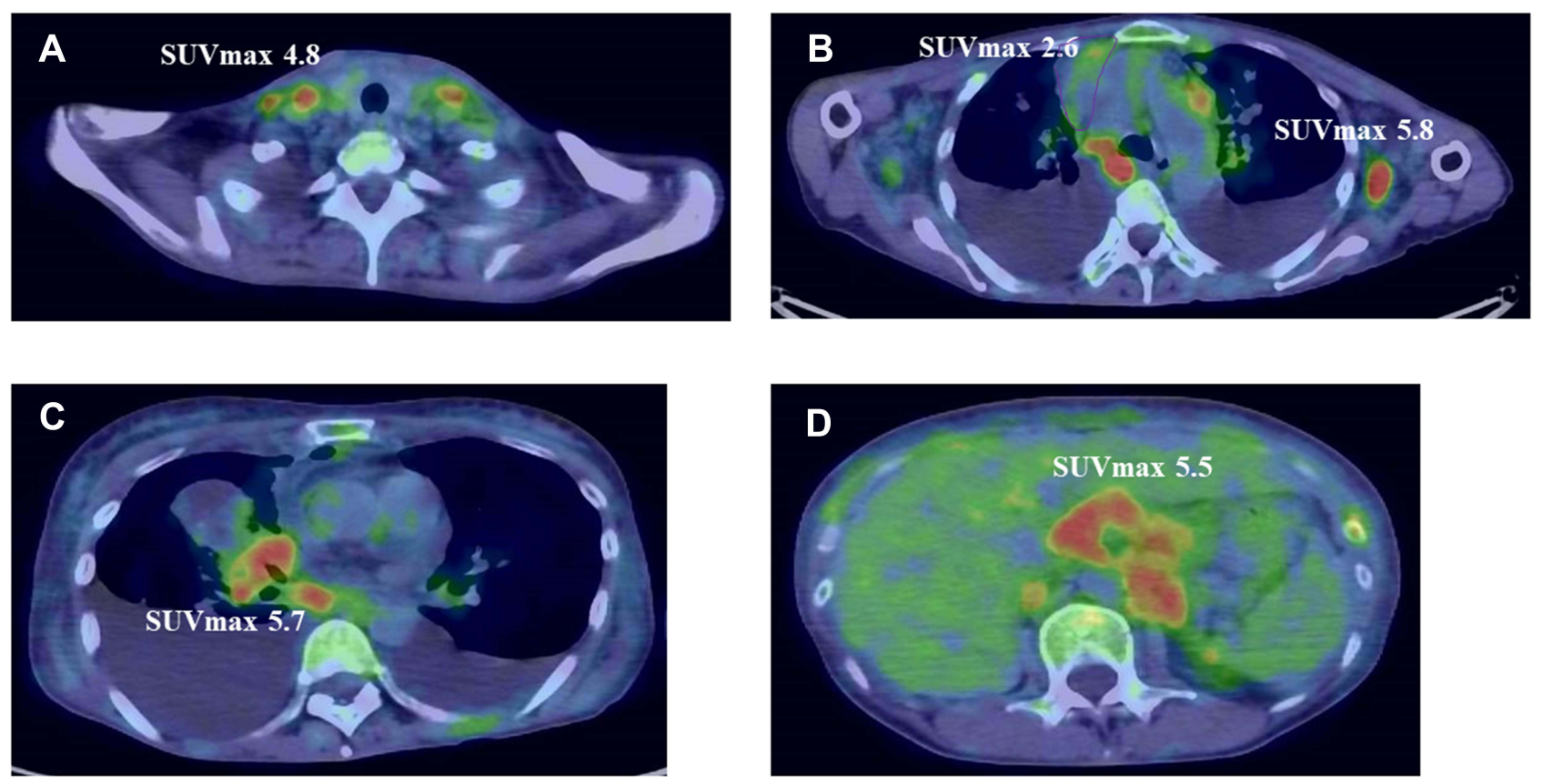

Figure I Fluorine-18 fluorodeoxyglucose positron emission tomography and computed tomography images of the patient at the time of diagnosis. Note bilateral uptake of fluorine-18 fluorodeoxyglucose in cervical (A), axillary (B), hilar (C), and abdominal para-aortic lymph nodes (D), in addition to a mass anterior mediastinum (B).

nodes throughout the body (Figure 1). High-resolution CT showed bronchiectasis in addition to multiple nodules consistent with lung metastases. CT-guided needle biopsy was performed to obtain tissue samples from the anterior mediastinal tumor. Hematoxylin/eosin staining of the collected specimens revealed a fascicular pattern composed primarily of spindle-shaped or oval epithelial cells. Immunohistochemical examination demonstrated that the sample was positive for cytokeratin AE1/AE3 and p40 (Figure 2), consistent with thymic epithelial tumor.
Concurrent serum tests revealed an sIL-2R level of 2774 $\mathrm{U} / \mathrm{mL}$ and hypogammaglobulinemia. Bone marrow biopsy demonstrated normal cells. Atypical epithelial cells were detected in the pleural effusion, consistent with thymic dissemination. Therefore, the patient was diagnosed with advanced type A thymoma (Masaoka stage IVb) and Good syndrome.

The patient received four cycles of the ADOC regimen, which comprised $40 \mathrm{mg} / \mathrm{m}^{2}$ doxorubicin and $50 \mathrm{mg} / \mathrm{m}^{2}$ cisplatin on day $1,0.6 \mathrm{mg} / \mathrm{m}^{2}$ vincristine on day 3 , and $700 \mathrm{mg} /$
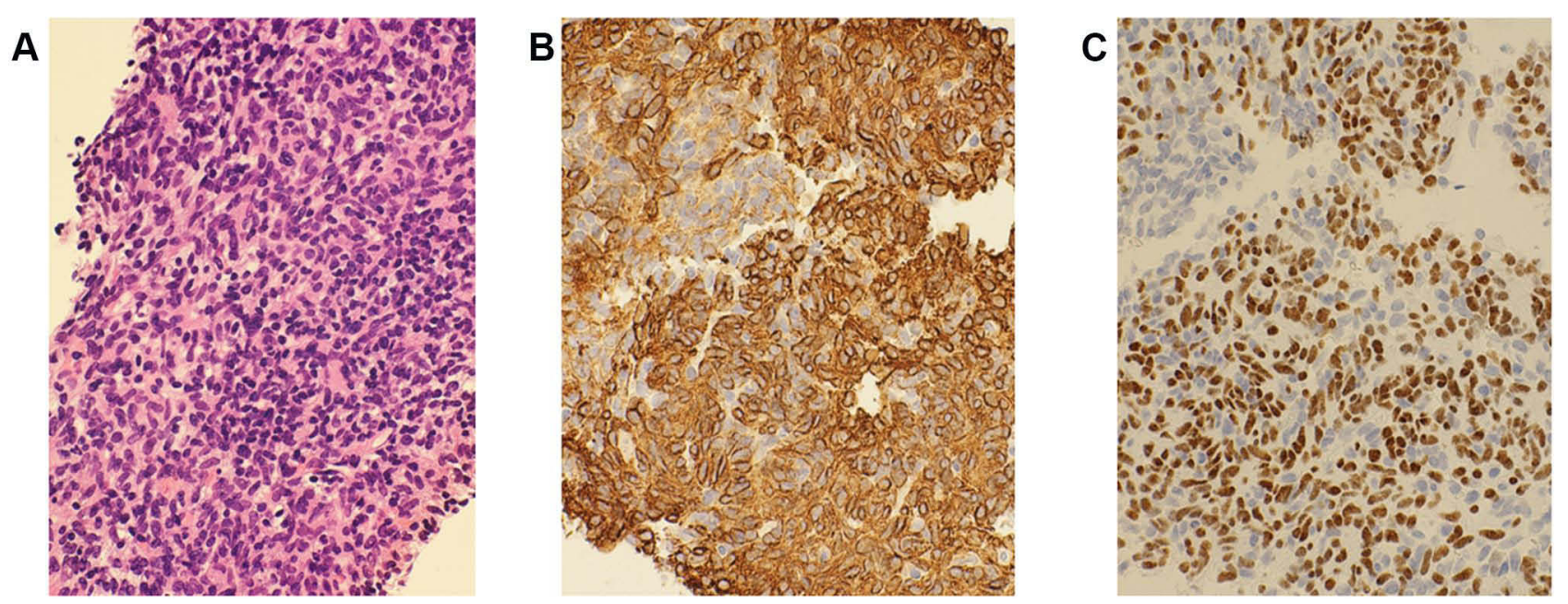

Figure 2 Histopathological examination of the anterior mediastinal tumor. Hematoxylin/eosin staining (A) shows a fascicular pattern composed primarily of spindle-shaped or oval epithelial cells. Cytokeratin AEI/AE3 (B) and p40 (C) immunostaining of the tumor specimen demonstrates a thymic epithelial tumor (magnification, $\times 400$ ). 

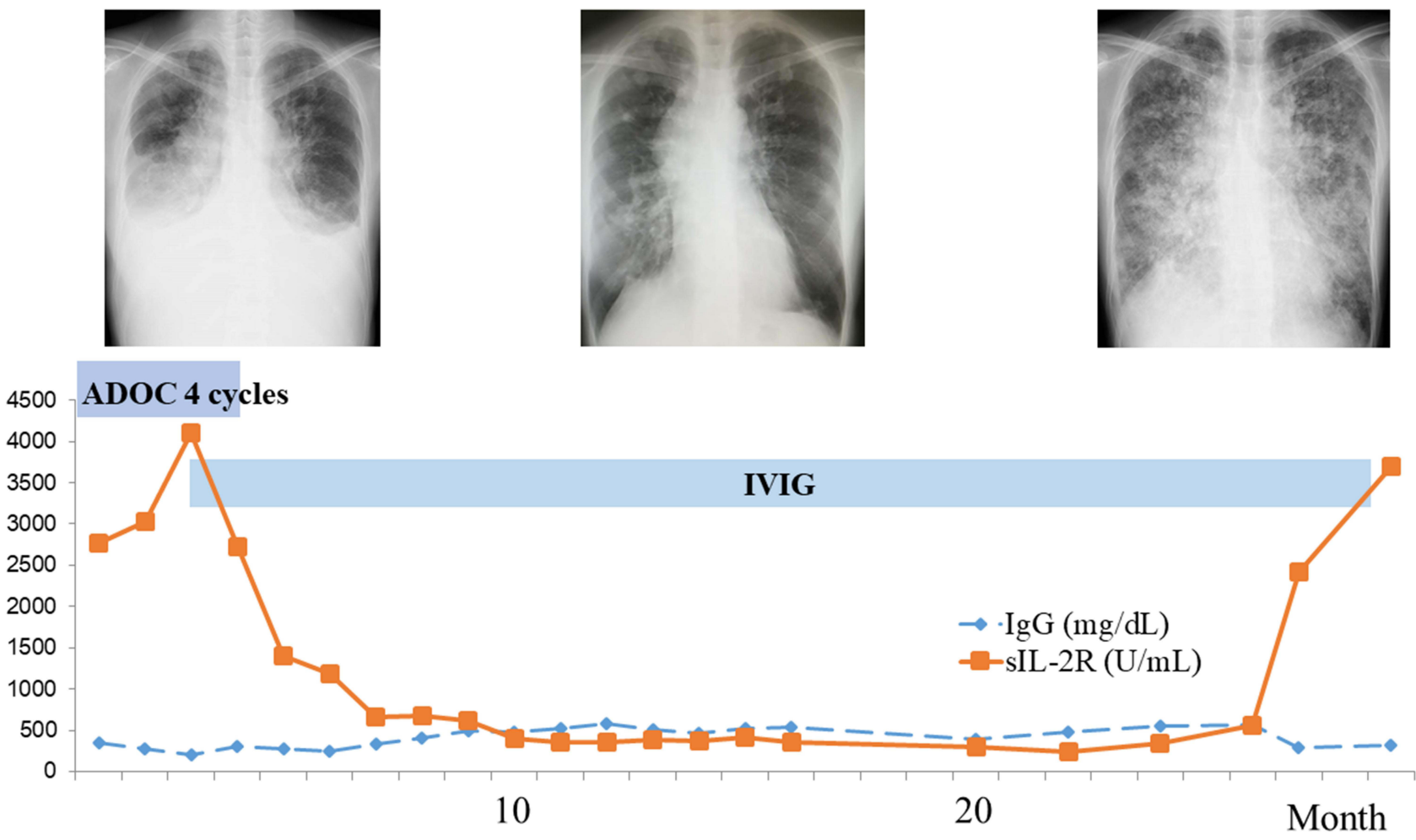

Figure 3 Clinical course. Top left, chest radiograph of the patient before chemotherapy. Top center, chest radiograph after chemotherapy, showing marked regression of both the tumors and the pleural effusion. Top right, last chest radiograph. Bottom, Line chart shows a reduction in the levels of soluble interleukin-2 receptor (sIL-2R) and a plateau in IgG levels following treatment with the ADOC combination chemotherapy regimen, comprising doxorubicin, cisplatin, vincristine, and cyclophosphamide, in combination with intravenous immunoglobulin.

$\mathrm{m}^{2}$ cyclophosphamide on day 4 . In addition, intravenous immunoglobulin was administered every four weeks. The size of the primary thymoma, systemic lymph node metastases, parenchymal lung metastases, and bilateral pleural effusion diminished in parallel to a decline in sIL-2R levels to the normal range (Figures 3 and 4). The patient achieved partial response with the ADOC chemotherapy according to the Response Evaluation Criteria in Solid Tumors version 1.1. ${ }^{6}$ Following a symptom-free period of 26 months, the patient died suddenly from pneumonia and autopsy revealed thymoma recurrence. Of note, the sIL-2R level was elevated in parallel to the clinical exacerbation (Figure 3).

\section{Discussion}

The present patient was diagnosed with type A thymoma based on the mediastinal tumor biopsy results and classified as stage IVb according to the Masaoka's criteria ${ }^{7}$ because of lymphogenous and hematogenous metastases. Complete surgical resection with or without chemotherapy is recommended for stage I-IVa, but not for stage IVb, thymoma. ${ }^{8}$ Cisplatin plus anthracycline is the most commonly used first-line chemotherapy for advanced thymoma., ${ }^{9,10}$ Thymectomy, which is reported to achieve a favorable effect on associated conditions such as myasthenia gravis and pure red blood cell aplasia, does not reverse hypogammaglobulinemia., ${ }^{2,11}$ In addition, myasthenia gravis and pure red blood cell aplasia respond to corticosteroid therapy in some cases, suggesting an autoimmune pathogenesis in thymoma. Corticosteroid treatment, in contrast, does not correct the immunodeficiency, ${ }^{2}$ consistent with the findings in the present patient who was treated with chemotherapy. In the present case, bronchiectasis was scattered in bilateral upper lobes, suggesting recurrent respiratory tract infection. Hypogammaglobulinemia is associated with mortality in patients with thymoma, ${ }^{2,3}$ and immunoglobulin replacement has been recognized as an effective approach to reduce the incidence of infections. ${ }^{12}$ Intravenous immunoglobulin should therefore be considered particularly for patients with Good syndrome and repeated infections. ${ }^{5}$

There are currently no validated biomarkers that can predict the prognosis of patients with thymoma. In the present case, the sIL-2R levels were elevated at the time of diagnosis and returned to within normal limits after chemotherapy. However, the sIL-2R levels ultimately increased with the recurrence of thymoma, and the patient 
A

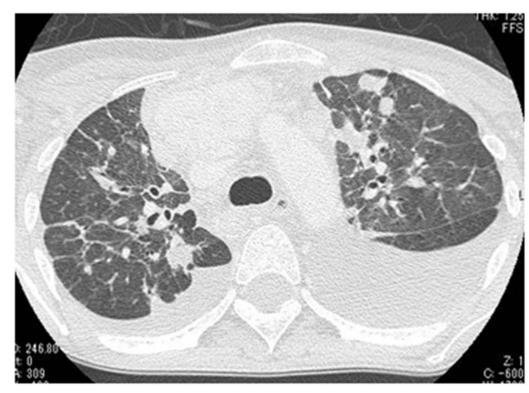

$\operatorname{IgG}(\mathrm{mg} / \mathrm{dL}) \quad 349$

$\operatorname{IgA}(\mathrm{mg} / \mathrm{dL}) \quad 73$

$\operatorname{IgM}(\mathrm{mg} / \mathrm{dL}) \quad<5$
B

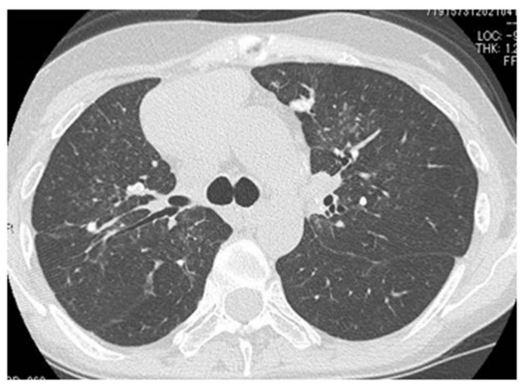

572

24

$<5$
C

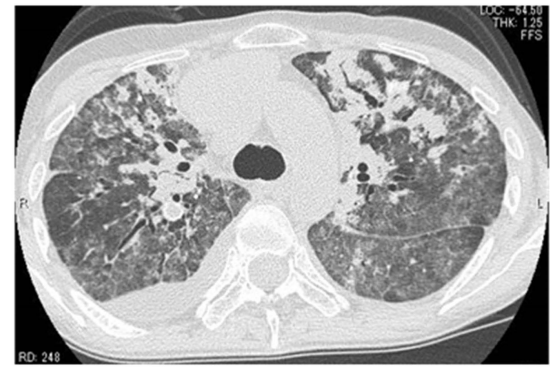

125

17

$<5$

Figure 4 Computed tomography images and immunoglobulin levels of the patient during the clinical course. Computed tomography image of the patient before chemotherapy $(\mathbf{A})$, after chemotherapy showing the best response (B), just prior to death $(\mathbf{C})$.

died. Thus, the changes in sIL-2R levels followed the patient's clinical course. IL-2R comprises three noncovalently associated proteins, IL-2R $\alpha$, IL-2R $\beta$, and IL-2R $\gamma \mathrm{c} .{ }^{13}$ Of the three chains, only IL-2R $\alpha$ is unique to IL- $2 R$. Although IL-2R $\beta$ and IL-2R $\gamma c$ are expressed in all lymphocytes including those that are dormant, IL- $2 \mathrm{R} \alpha$ is detected only after lymphocyte activation. T lymphocyte activation by antigens and costimulators leads to the expression of the IL-2R $\alpha$ chain. Thus, chronic $\mathrm{T}$ lymphocyte stimulation leads to IL-2R $\alpha$ secretion and increased levels of IL- $2 \mathrm{R} \alpha$ shed in serum, namely sIL-2R, is considered as a clinical marker of strong antigenic stimulation, such as that may occur in hematopoietic malignancies. ${ }^{14-16}$ Increased serum sIL-2R is also considered as an index of immune-related adverse events caused by immune checkpoint inhibitors. ${ }^{17}$ Multiple studies reported immunological diversity in Good syndrome. ${ }^{2,5,18-20}$ In addition to hypogammaglobulinemia, other immunological features of Good syndrome include reduction in peripheral $\mathrm{B}$ cell count, $\mathrm{CD} 4+$ $\mathrm{T}$ lymphopenia, inverted CD4/CD8 ratio, and reduced $\mathrm{T}$ lymphocyte proliferative response to mitogen stimulation. T lymphocytes might escape normal maturation and produce insufficient levels of sIL-2R in some patients with Good syndrome, whereas the current patient did not exhibit any bone marrow abnormalities, indicating an intact cellular immunity and sufficient sIL-2R production. In summary, the current case suggests that sIL-2R might be a useful prognostic biomarker in a subset of patients with Good syndrome.

\section{Ethical Approval}

Official approval for the study was obtained in advance from the Ethics Committee at Showa University (approved number 269).

\section{Patient Consent for Publication}

The patient provided written informed consent.

\section{Acknowledgment}

The authors are grateful to Mari Nakamoto, Saori Kawamura, Miku Kosuge, Hidekazu Cho, Shohei Shimizu, Akira Fujishima, Ayaka Mase, Yuki Osakabe, Toshitaka Funaki, Daisuke Inoue, Yohei Yamazaki, Hidetsugu Tateno, Takuya Yokoe for their assistance in the interpretation of the results and critical review of the manuscript.

\section{Author Contributions}

All authors examined and cared for the patient. All authors contributed to data analysis, drafting or revising the article, have agreed on the journal to which the article will be submitted, gave final approval of the version to be published, and agree to be accountable for all aspects of the work. 


\section{Disclosure}

The authors declare no conflicts of interest in connection with this paper, and received no payment or services from a third party in relation to this study.

\section{References}

1. Regnard JF, Magdeleinat P, Dromer C, et al. Prognostic factors and long-term results after thymoma resection: a series of 307 patients. J Thorac Cardiovasc Surg. 1996;112:376-384. doi:10.1016/S00225223(96)70265-9

2. Kelesidis T, Yang O. Good's syndrome remains a mystery after 55 years: a systematic review of the scientific evidence. Clin Immunol. 2010;135:347-363. doi:10.1016/j.clim.2010.01.006

3. Jansen A, van Deuren M, Miller J, et al. Prognosis of Good syndrome: mortality and morbidity of thymoma associated immunodeficiency in perspective. Clin Immunol. 2016;171:12-17. doi:10.1016/j. clim.2016.07.025

4. Scorsetti M, Leo F, Trama A, et al. Thymoma and thymic carcinomas. Crit Rev Oncol Hematol. 2016;99:332-350. doi:10.1016/j. critrevonc.2016.01.012

5. Kelleher P, Misbah SA. What is Good's syndrome? Immunological abnormalities in patients with thymoma. $J$ Clin Pathol. 2003;56:12-16. doi:10.1136/jcp.56.1.12

6. Eisenhauer EA, Therasse P, Bogaerts J, et al. New response evaluation criteria in solid tumours: revised RECIST guide- line (version 1.1). Eur J Cancer. 2009;45:228-247. doi:10.1016/j.ejca.2008.10.026

7. Masaoka A. Staging system of thymoma. J Thorac Oncol. 2010;5: S304-S312. doi:10.1097/JTO.0b013e3181f20c05

8. Falkson CB, Bezjak A, Darling G, et al. The management of thymoma: a systematic review and practice guideline. J Thorac Oncol. 2009;4:911-919. doi:10.1097/JTO.0b013e3181a4b8e0

9. Berghmans T, Durieux V, Holbrechts S, et al. Systemic treatments for thymoma and thymic carcinoma: a systematic review. Lung Cancer. 2018;126:25-31. doi:10.1016/j.lungcan.2018.10.018
10. Venuta F, Rendina EA, Anile M, de Giacomo T, Vitolo D, Coloni GF. Thymoma and thymic carcinoma. Gen Thorac Cardiovasc Surg. 2012;60:1-12. doi:10.1007/s11748-011-0814-0

11. Narahari NK, Gongati PK, Uppin SG, Kapoor A, Kakarla B, Tella RD. A 66-year-old man with mediastinal mass and dyspnea. Chest. 2016;150:e109-15. doi:10.1016/j.chest.2016.07.002

12. Tarr PE, Sneller MC, Mechanic LJ, et al. Infections in patients with immunodeficiency with thymoma (Good syndrome). Report of 5 cases and review of the literature. Medicine. 2001;80:123-133. doi:10.1097/00005792-200103000-00005

13. Witkowska AM. On the role of sIL-2R measurements in rheumatoid arthritis and cancers. Mediators Inflamm. 2005;14:121-130. doi:10.1155/MI.2005.121

14. Chrobák L. Clinical significance of soluble interleukin-2 receptor. Acta Med. 1996;39:3-6.

15. Kusano Y, Yokoyama M, Terui Y, et al. High pretreatment level of soluble interleukin-2 receptor is a robust prognostic factor in patients with follicular lymphoma treated with R-CHOP-like therapy. Blood Cancer J. 2017;7:e614. doi:10.1038/bcj.2017.96

16. Murakami J, Arita K, Wada A, et al. Serum soluble interleukin-2 receptor levels for screening for malignant lymphomas and differential diagnosis from other conditions. Mol Clin Oncol. 2019;11:474-482. doi:10.3892/mco.2019.1922

17. Takai R, Funakoshi Y, Suto H, et al. Serum soluble interleukin-2 receptor as a potential biomarker for immune-related adverse events. Anticancer Res. 2021;41:1021-1026. doi:10.21873/anticanres.14857

18. Arnold SJ, Hodgson T, Misbah SA, Patel SY, Cooper SM, Venning VA. Three difficult cases: the challenge of autoimmunity, immunodeficiency and recurrent infections in patients with Good syndrome. Br J Dermatol. 2015;172:774-777. doi:10.1111/bjd.13293

19. Martinez B, Browne SK. Good syndrome, bad problem. Front Oncol. 2014;4:307. doi:10.3389/fonc.2014.00307

20. Kawamura T, Naito T, Kobayashi H, et al. Acquired immunodeficiency associated with thymoma: a case report. BMC Cancer. 2019;19:762. doi:10.1186/s12885-019-5980-y
OncoTargets and Therapy

\section{Publish your work in this journal}

OncoTargets and Therapy is an international, peer-reviewed, open access journal focusing on the pathological basis of all cancers, potential targets for therapy and treatment protocols employed to improve the management of cancer patients. The journal also focuses on the impact of management programs and new therapeutic agents and protocols on patient perspectives such as quality of life, adherence and satisfaction. The manuscript management system is completely online and includes a very quick and fair peer-review system, which is all easy to use. Visit http://www.dovepress.com/ testimonials.php to read real quotes from published authors. 